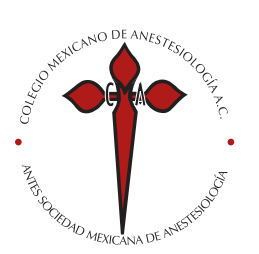

\section{Analgesia obstétrica en la paciente con síndrome de Stickler: un reporte de caso y revisión de la literatura}

\author{
Obstetric analgesia in the patient with Stickler syndrome: \\ a case report and review of the literature
}

Dr. Ulises Sánchez-Vásquez, * Dra. Mariela García-Bravo, ${ }^{\ddagger}$ Dra. Giselle Zumaya-Mancilla,§ Dra. Xóchitl Popoca-Mondragón, " Dr. Marco Antonio Chávez-Ramírez"

Citar como: Sánchez-Vásquez U, García-Bravo M, Zumaya-Mancilla G, Popoca-Mondragón X, Chávez-Ramírez MA. Analgesia obstétrica en la paciente con síndrome de Stickler: un reporte de caso y revisión de la literatura. Rev Mex Anestesiol. 2021; 44 (2): 139-142. https://dx.doi.org/10.35366/99018

RESUMEN. El síndrome de Stickler tiene una incidencia de uno en 7,500-9,000 recién nacidos. Es una enfermedad del tejido conectivo con patrón de herencia autosómico dominante en la mayoría de los casos, aunque también con patrón recesivo, por afección patogénica de genes procolágeno como COL2A1, COL11A1, entre otros. Cursa con manifestaciones multisistémicas craneofaciales, oculares, auditivas, esqueléticas/articulares y se ha asociado a prolapso de la válvula mitral. Se diferencian tres tipos: tipo I (el más frecuente, 75\%) o síndrome de Stickler completo, tipo II o síndrome de Stickler ocular y el tipo III u oto-espóndilo-displasia megaepifisaria. El diagnóstico se realiza al probar las manifestaciones 0 al demostrar una variante genética patogénica. Respecto al manejo anestésico en la paciente obstétrica, se recomienda evitar la anestesia general. Se presenta el caso de una paciente de 26 años con diagnóstico de síndrome de Stickler tipo I, con embarazo a término y su relación con la anestesia neuroaxial. A pesar de su incidencia, no existe literatura médica acerca del manejo de la anestesia neuroaxial en este tipo de pacientes.

ABSTRACT. Stickler syndrome has an incidence of 1/7.500-9.000 in newborns. It is a connective tissue disease with an autosomal dominant inheritance pattern in most cases, but also with a recessive pattern, due to the pathogenic involvement of procollagen genes such as COL2A1, COL11A1, among others. It is characterized by craniofacial, ocular, auditory and skeletal/joint multisystemic signs and symptoms, and has been associated with prolapse of the mitral valve. Three types are differentiated: type I (the most frequent, 75\%) or complete Stickler syndrome, type II or ocular Stickler syndrome and type III or oto-espondilo-megaepiphyseal dysplasia. The diagnosis is made by confirming the signs and symptoms or by verifying the presence of a pathogenic genetic variant. With respect to the anesthetic management in the obstetric patient, it is recommended to avoid general anesthesia. The article illustrates the case of a female patient of 26 years with Stickler syndrome type I and full-term pregnancy, and associated implications for neuroaxial anesthesia. Despite its incidence, there is no medical literature on the management of neuraxial anesthesia in this type of patients.

\section{INTRODUCCIÓN}

$\mathrm{E}$ l síndrome de Stickler (SS), también conocido como artrooftalmopatía progresiva hereditaria o síndrome de Marshall-Stickler, es una condición médica rara causada por mutaciones de las cadenas alfa de la colágena tipo 2 y 11, con disrupción subsecuente de los tejidos conectivos y otras alteraciones multisistémicas ${ }^{(1)}$. Tiene una prevalencia de uno en cada 7,500-9,000 recién nacidos, por lo que es la enfermedad hereditaria del tejido conectivo más común ${ }^{(2)}$.

Fue descrito por primera vez en 1965 por Gunnar Stickler ${ }^{(3)}$, en la mayoría de los casos sigue un patrón de herencia autosómico dominante y es causado por variantes patogénicas de COL2A1, COL11A1 o COL11A2, con 50\% de probabilidades de ser heredado, aunque al seguir un patrón recesivo se asocia a las variantes patogénicas de COL9A1, COL9A2 o COL9A3,
Palabras clave:

Síndrome de Stickler, anestesia neuroaxial, embarazo, vía aérea difícil.

Keywords:

Stickler syndrome, neuraxial anesthesia, pregnancy, difficult airway.

* Anestesiólogo, Centro Médico ABC.

‡ Médico residente de Anestesiología, Centro Médico ABC.

$\S$ Anestesiólogo, Instituto

Nacional de Perinatología.

^ Anestesiólogo, Hospital

Juárez de México.

\| Jefe Corporativo de Anestesiología, Centro Médico ABC.

Ciudad de México, México.

Correspondencia:

Dr. Ulises Sánchez-Vásquez

The American British Cowdray, Medical Center, IAP

Sur 136 Núm. 116,

Col. Las Américas, Alcaldía Álvaro Obregón, 01120, Ciudad de México. Tel. 5549532782

E-mail: ulises.sanchez. vasquez@gmail.com

Recibido: 16-07-2019 Aceptado:04-09-0019

Abreviaturas: $\mathrm{CP}=$ Catéter peridural . PVM = Prolapso de la válvula mitral. SPR = Secuencia de Pierre-Robin. SS = Síndrome de Stickler. 
con $25 \%$ de probabilidades de ser transmitido, $50 \%$ de ser portador asintomático y $25 \%$ de no ser afectado $^{(4)}$.

Se subdivide en tres tipos cuando es inherente a un patrón autosómico dominante, y en otros cuando presenta una herencia autosómica recesiva:

- Tipo 1, artrooftalmopatía progresiva hereditaria o SS completo: es el más común, se presenta en $80-90 \%$ de los casos. Se debe a una mutación en el gen procolágeno COL2A1.

- Tipo 2 o SS ocular: la mutación ocurre en COL11A1 y se presenta en $10-20 \%$ de los casos.

- Tipo 3 oto-espóndilo-displasia megaepifisaria o SS noocular: se debe a mutación en COL11A2.

- Otros: Se debe a mutaciones en COL9A1, COL9A2, COL9A3 y LOXL3 ${ }^{(2,4)}$.

La colágena tipo 2 se localiza principalmente en el cartílago hialino y en el humor vítreo, de ahí sus manifestaciones clíni$\operatorname{cas}^{(5)}$. Es una enfermedad multisistémica con manifestaciones oculares (miopía, catarata, desprendimiento de retina), auditivas (sordera neurosensorial y/o de conducción), craneofaciales (hipoplasia malar, micro/retrognatia y paladar hendido) y esqueléticas/articulares (escoliosis, cifosis, espondilolistesis, displasia espondiloepifisaria leve y artritis precoz) ${ }^{(6)}$. Este síndrome no se asocia a limitaciones $\operatorname{cognitivas}^{(4)}$. Se estima que un tercio de los pacientes con secuencia de Pierre-Robin (SPR) tienen $\mathrm{SS}^{(5,7)}$. En un estudio realizado por Stickler y colaboradores en 612 personas con SS se encontraron las siguientes manifestaciones $^{(8)}$ (Tabla 1).

Anteriormente, el SS se llegó a asociar a prolapso de la válvula mitral $(\mathrm{PVM})^{(9)}$. Sin embargo, en un estudio reciente en pacientes con diagnóstico molecular establecido se sugiere

\begin{tabular}{|c|c|c|}
\hline $\mathrm{n}$ & Afección & Manifestaciones clínicas (\%) \\
\hline \multirow[t]{3}{*}{95} & Ocular & Miopía (90) \\
\hline & & Desprendimiento de retina (60) \\
\hline & & Ceguera (4) \\
\hline \multirow[t]{4}{*}{90} & Osteoarticulares & Hipermovilidad de las articulaciones (55) \\
\hline & & Genu valgum (25) \\
\hline & & Escoliosis (17) \\
\hline & & Pectus excavatum/carinatum (9) \\
\hline \multirow[t]{3}{*}{84} & Craneofacial & Hipoplasia mediofacial (52) \\
\hline & & Micrognatia (47) \\
\hline & & Paladar hendido (41) \\
\hline 70 & Auditiva & Sordera \\
\hline
\end{tabular}

Las manifestaciones clínicas del síndrome de Stickler incluyen alteraciones craneofaciales que predisponen a una vía aérea difícil así como alteraciones osteoarticulares que pueden dificultar el bloqueo neuroaxial. que tienen la misma prevalencia para esta afección que la población en general ${ }^{(10)}$. Al no contar con literatura concluyente se sugiere una evaluación cardiológica completa.

No existe un consenso sobre criterios diagnósticos, por ende, el diagnóstico se hace probando los datos clínicos antes mencionados y/o demostrando una variante genética patogénica ${ }^{(5)}$. La información sobre el manejo anestésico es escasa. Debido a que en estos pacientes se prevé una vía aérea difícil por las alteraciones craneofaciales, se recomienda la anestesia neuroaxial y/o regional en ausencia de contraindicación ${ }^{(11)}$. Respecto al manejo en la paciente embarazada con SS, se recomienda evitar la anestesia general ${ }^{(12)}$ por el mismo motivo, aunado a los cambios fisiológicos propios del embarazo ${ }^{(13)}$. A pesar de ser la conectivopatía hereditaria más común, no existe literatura médica relacionada a la anestesia neuroaxial en este tipo de pacientes.

\section{CASO CLÍNICO}

Paciente femenino de 26 años, nulípara, con diagnóstico de embarazo de 40.2 semanas de gestación y síndrome de Stickler tipo I, diagnosticado en la niñez. Referida al Instituto Nacional de Perinatología (INPer) por presentar dolor tipo obstétrico. Se nos interconsulta por analgesia obstétrica para atención del trabajo de parto.

A la exploración física talla $1.68 \mathrm{~m}$ y peso $88 \mathrm{~kg}$ (IMC $31.1 \mathrm{~kg} / \mathrm{m}^{2}$ ), se evidencian alteraciones craneofaciales como hipoplasia malar y micrognatia, implantación baja de orejas e hipoacusia bilateral con presencia de dispositivo coclear así como miopía. Precordio rítmico, sin fenómenos agregados y movimientos respiratorios limitados. En la columna dorsal se observa rotoescoliosis grave (Figura 1). A la valoración de la vía aérea se observa Mallampati III, distancia tiromentoniana $<6.5 \mathrm{~cm}$, movilidad cervical grado II según Bellhouse-Dore, protrusión mentoniana grado III, con apertura oral $>3 \mathrm{~cm}$. Paraclínicos sin contraindicaciones para la anestesia neuroaxial.

Paciente en sala de labor. Se coloca en decúbito lateral izquierdo, previa monitorización de signos vitales con TA 120/74 mmHg, FC 88 lpm, FCF 150 lpm, FR 20 rpm y SpO $93 \%$. Se colocan puntas nasales con $\mathrm{O}_{2}$ a $3 \mathrm{~L} / \mathrm{min}$. Se realiza asepsia y antisepsia de región lumbar y se colocan campos estériles. Se palpa trayecto escoliótico de columna vertebral y se identifica espacio intervertebral L2-L3, se infiltran piel y tejidos blandos con lidocaína simple al 2\% (3 mL/60 mg). Tras dos intentos, se llega a espacio peridural con aguja tipo Tuohy 17G mediante técnica de pérdida de la resistencia, y se administra dosis prueba sin alteraciones hemodinámicas; se instala catéter peridural (CP) cefálico sin incidentes. Se administra por CP dosis de ropivacaína $0.2 \%$ (20 mg/10 mL) y fentanilo $100 \mu \mathrm{g}$. Se reposiciona a paciente en semifowler. Queda paciente en labor para atención de trabajo de parto con monitoreo continuo y vigilancia clínica. 


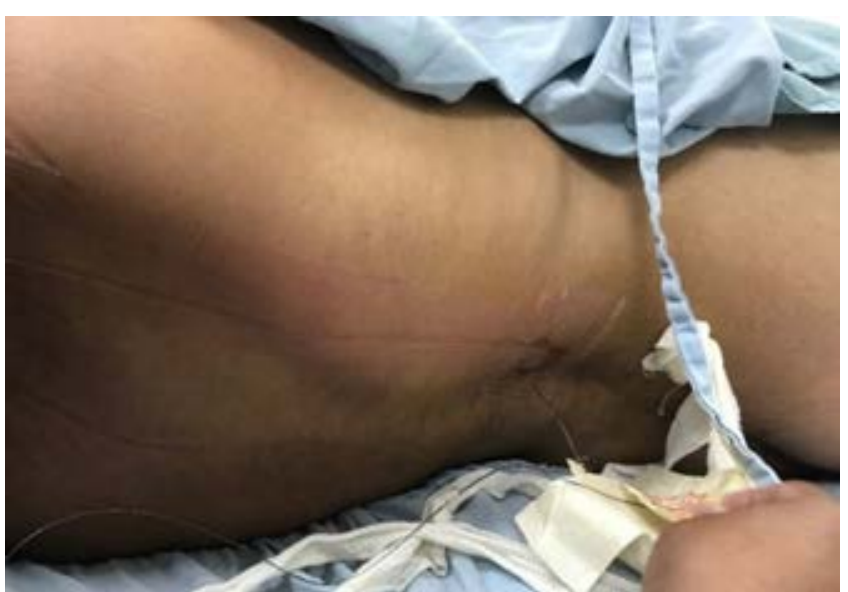

Figura 1: Trayecto escoliótico en paciente con síndrome de Stickler. Las alteraciones osteoarticulares incluyen la escoliosis que predispone a dificultades para la técnica neuroaxial.

Posteriormente, ingresa a sala de expulsión para atención de parto instrumentado con fórceps Simpson, bajo bloqueo peridural. Se realiza monitorización con EKG (DII, V5), PANI y pulsioxímetro. Se corrobora permeabilidad de CP y se administra dosis de rescate de lidocaína $2 \%$ con epinefrina $200 \mathrm{mg} / 10 \mathrm{~mL}$ hasta alcanzar una dosis total fraccionada de $500 \mathrm{mg} / 30 \mathrm{~mL}$ y fentanilo $150 \mu \mathrm{g}$ peridurales. Se mantiene ventilación espontánea con $\mathrm{O}_{2}$ por puntas nasales a $3 \mathrm{~L} / \mathrm{min}$. Se obtiene producto único vivo de sexo femenino, peso 3,225 g, talla 49 cm, Apgar 8/9, Silverman 2, Capurro 40.4 semanas de gestación. Egresos de $960 \mathrm{~mL}$ (sangrado $200 \mathrm{~mL}$ y diuresis $200 \mathrm{~mL}$ ) e ingresos de 1,000 mL con balance hídrico positivo de $40 \mathrm{~mL}$. Misceláneos: oxitocina 12.5 UI IV. Se concluye procedimiento sin incidentes. Se retira CP íntegro y completo. Pasa a recuperación con TA 112/60 mmHg, FC 79 lpm, $\mathrm{SpO}_{2}$ 100\%, Bromage III y Aldrete 9.

\section{DISCUSIÓN}

Debido a las alteraciones craneofaciales aunadas a los cambios fisiológicos propios del embarazo, el SS se asocia a una vía aérea difícil predicha ${ }^{(8,13)}$, por lo que el manejo anestésico recomendado es evitar la anestesia general por las dificultades que se pueden encontrar al abordar la vía aérea ${ }^{(12)}$. Nuestra paciente fue manejada mediante bloqueo peridural debido a que la atención del trabajo de parto así lo requiere y por lo previamente señalado. El bloqueo fue exitoso al segundo intento; sin embargo, se encontraron dificultades a la palpación del trayecto espinal y de los espacios intervertebrales.

Nuestra paciente presentaba características clínicas del SS tipo I descrito por Peter S. Rose y colaboradores ${ }^{(6)}$. Las consideraciones anestésicas incluyeron la posibilidad de una vía aérea difícil por las alteraciones craneofaciales, por lo que se idearon planes alternos en caso de requerir abordar de manera urgente la vía aérea así como la posibilidad de que la paciente presentara $\mathrm{PVM}^{(10)}$, aunque no se encontró ninguna anormalidad cardíaca a la auscultación ni en la valoración ecocardiográfica de nuestra paciente.

La valoración preanestésica debe ser minuciosa al valorar la vía aérea, nuestra paciente presentó indicadores positivos de una vía aérea difícil predicha, por lo que se tomaron precauciones necesarias como contar con la posibilidad de un carro de vía aérea difícil a pesar de que el abordaje no incluía manejar la vía aérea, ya que existe la posibilidad de reacciones adversas en la anestesia neuroaxial como un alto bloqueo o una raquia masiva que requieren del abordaje urgente de la vía aérea.

Las alteraciones osteoarticulares, incluidas las de la columna dorsal, están reportadas hasta en $90 \%{ }^{(8)}$, por lo que se aconsejan métodos como ultrasonografía para garantizar el éxito de la técnica neuroaxial ${ }^{(14)}$. Hay que recordar que la colocación del CP es una técnica a ciegas que deriva en situaciones en las que hay variaciones en la posición de la punta del catéter o en la disfunción de éste ${ }^{(15,16)}$, lo anterior es una posible justificación de la dosis alta del anestésico local que requirió nuestra paciente, aunque es importante aclarar que se evaluaron datos de toxicidad sin encontrarse ninguno.

Cork y colaboradores demostraron por primera vez la utilidad de la ecografía de la columna lumbar para guiar los procedimientos neuroaxiales ${ }^{(17)}$. En la década de 2000, Grau y su equipo expandieron significativamente la investigación en el campo e introdujeron la visualización en el plano sagital paramediano $^{(18)}$. El rendimiento de la anestesia y analgesia neuroaxial puede ser un desafío único en la paciente obstétrica ${ }^{(19)}$, aunado a alteraciones anatómicas propias de un síndrome con alteraciones osteoarticulares como el caso de nuestra paciente. Existen descripciones detalladas sobre la técnica, el momento de la evaluación ultrasonográfica se realiza inmediatamente antes del procedimiento neuroaxial para identificar puntos de referencia y se recomienda un transductor curvo de baja frecuencia (2-5 MHz). Se ha demostrado la superioridad del ultrasonido para identificar correctamente el nivel vertebral al ser comparado con técnicas radiológicas como la radiografía lateral lumbar, la resonancia magnética y la tomografía computarizada así como la palpación, resultando en una tasa de éxito general más elevada ${ }^{(20)}$. Por tal motivo, ante alteraciones anatómicas de la columna se recomienda su uso para garantizar el bloqueo neuroaxial, además el hecho de carecer de radiación lo hace ideal para la paciente obstétrica.

Un panel de expertos de la Sociedad Americana de Anestesia Regional y Medicina del Dolor (ASRA) concluyó recientemente que existe evidencia de nivel Ia que respalda que la ecografía neuroaxial aumenta la eficiencia de la anestesia neuroaxial lumbar, incluso en pacientes técnicamente difíciles como en la escoliosis ${ }^{(21)}$. Es importante mencionar que la escoliosis idiopática tiene una prevalencia reportada de 
hasta 5.2\% en la población en general con aproximadamente el doble de mujeres afectadas ${ }^{(22)}$. Así la ecografía es útil para localizar los procesos espinosos, identificar la dirección de la asimetría de los procesos articulares, determinar la profundidad, y seleccionar el punto y el ángulo óptimos para la entrada de la aguja ${ }^{(20)}$.

\section{CONCLUSIONES}

La valoración preanestésica debe hacerse de manera minuciosa en búsqueda de predictores de vía aérea difícil y de posibles alteraciones cardíacas como PVM, por lo que se aconseja una valoración clínica completa con paraclínicos, ya sea electrocardiograma o ecocardiografía así como la formulación de planes para el abordaje de la vía aérea en casos urgentes. Ante una vía aérea difícil predicha debe considerarse la intubación endotraqueal con fibroendoscopio flexible cuando se decida anestesia general. Cuando se prefiera anestesia regional es importante tomar en cuenta que esta población puede presentar alteraciones esqueléticas en la columna vertebral que pueden dificultar el bloqueo, y que el ultrasonido es una herramienta no invasiva que aumenta las probabilidades de un bloqueo exitoso.

\section{REFERENCIAS}

1. Varandas J, Brito S, Rodrigues M, Cavaleiro C, Pimenta A, Machado HS. Airway management in stickler syndrome patient: a case report. J Anesth Clin Res. 2017;8:2.

2. Weith T, Münster T, Prottengeier J. OrphanAnesthesia-Getting the key points right for anaesthesia in patients with rare diseases. Trends Anaesth Crit Care. 2018;22:22-25.

3. Stickler G. Hereditary progressive arthro-ophthalmopathy II: additional observation on vertebral abnormalities, a hearing defect, and a report of a similar case. Mayo Clin Proc. 1967;42:495-500.

4. Robin NH, Moran RT, Ala-Kokko L. Stickler syndrome. GeneReviews $\left({ }^{\circledR}\right)$. 2017;5:100-130.

5. Antunes RB, Alonso N, Paula RG. Importance of early diagnosis of Stickler syndrome in newborns. J Plast Reconstr Aesthetic Surg. 2012;65:1029-1034.

6. Rose PS, Ahn NU, Levy HP, Ahn UM, Davis J, Liberfarb RM, et al. Thoracolumbar spinal abnormalities in Stickler syndrome. Spine (Phila Pa 1976). 2001;26:403-409.

7. Holder-Espinasse M, Abadie V, Cormier-Daire V, Beyler C, Manach Y, Munnich A, et al. Pierre Robin sequence: a series of 117 consecutive cases. J Pediatr. 2001;139:588-590.

8. Stickler GB, Hughes W, Houchin P. Clinical features of hereditary progressive arthroophthalmopathy (Stickler syndrome): A survey. Genet Med. 2001;3:192-196.

9. Liberfarb RM, Goldblatt A. Prevalence of mitral valve prolapse in the Stickler syndrome. Am J Med Genet. 1986;24:387-392.

10. Ahmad N, Richards AJ, Murfett HC, Shapiro L, Scott JD, Yates JR, Snead MP. Prevalence of mitral valve prolapse in the Stickler syndrome. Am J Med Genet Part A. 2003;116:234-237.

11. Kücükyavuz Z, Ozkaynak O, Tüzüner AM, Kisnisci R. Difficulties in anesthetic management of patients with micrognathia: report of a patient with Stickler syndrome. Oral Surgery, Oral Med Oral Pathol Oral Radiol Endodontology. 2006;102:33-36.
12. Heisnam I, Singh S, Singh K, Singh P, Singh I. Stickler syndrome: anaesthetic considerations - a case report. J Evol Med Dent Sci. 2012;1:343-347.

13. Topulos GP, Datta S, Chandrasekhar S, Kodali B-S, Bulich LN. Airway changes during labor and delivery. Anesthesiology. 2009;108:357-362.

14. Carrillo-Esper R, Ponce-Medrano JAD, Huesca-Jiménez G, IbariasEnciso IA, Garnica-Escamilla MA, Cruz-Gutiérrez EO. Ultrasonografía del espacio epidural lumbar. Rev Mex Anestesiol. 2017;40:134-137.

15. Myers LB, Bulich LA. Anesthesia for ex utero intrapartum treatment exit procedure. In: Myers LB, Bulich LA. Anesthesia for fetal intervention and surgery. Hamilton, Ontario: Editorial BC Decker Inc.; 2005. pp. 145-156.

16. López U, Bermúdez A, Solares A, Fuentes R, Aguirre L. Corroboración radiológica de catéteres peridurales instalados crónicamente en pacientes con dolor. Rev Mex Anestesiol. 2001;24.

17. Corr RC, Kryc JJ, Vaughan RW. Ultrasonic localization of the lumbar epidural space. Anesthesiol J Am Soc Anesthesiol. 1980;52:513-515.

18. Grau T, Leipold RW, Conradi R, Martin E. Ultrasound control for presumed difficult epidural puncture. Acta Anaesthesiol Scand. 2001;45:766-771.

19. Grau T, Leipold RW, Conradi R, Martin E, Motsch J. Efficacy of ultrasound imaging in obstetric epidural anesthesia. J Clin Anesth. 2002;14:169-175.

20. Lee A, Loughrey JPR. The role of ultrasonography in obstetric anesthesia. Best Pract Res Clin Anaesthesiol. 2017;31:81-90.

21. Neal JM, Brull R, Horn J, Liu SS, McCartney CJL, Perlas A, et al. The second american society of regional anesthesia and pain medicine evidence-based medicine assessment of ultrasound-guided regional anesthesia: executive summary. Reg Anesth Pain Med. 2016;41:181-194.

22. Konieczny MR, Senyurt H, Krauspe R. Epidemiology of adolescent idiopathic scoliosis. J Child Orthop. 2012;7:3-9. 